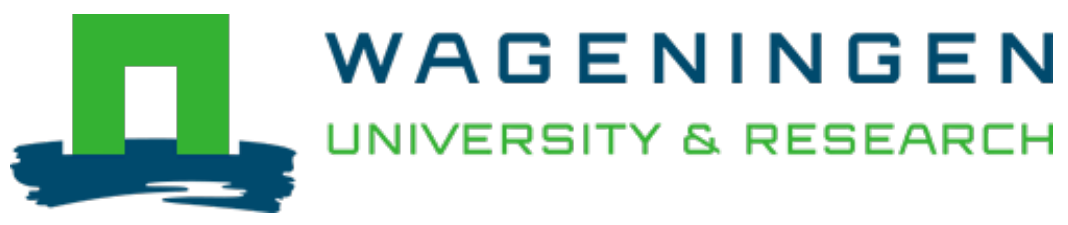

\title{
Environmental Control of Amyloid Polymorphism by Modulation of Hydrodynamic Stress
}

\author{
ACS Nano \\ Zhou, Jiangtao; Venturelli, Leonardo; Keiser, Ludovic; Sekatskii, Sergey K.; Gallaire, François et al \\ https://doi.org/10.1021/acsnano.0c07570
}

This article is made publicly available in the institutional repository of Wageningen University and Research, under the terms of article $25 \mathrm{fa}$ of the Dutch Copyright Act, also known as the Amendment Taverne. This has been done with explicit consent by the author.

Article 25 fa states that the author of a short scientific work funded either wholly or partially by Dutch public funds is entitled to make that work publicly available for no consideration following a reasonable period of time after the work was first published, provided that clear reference is made to the source of the first publication of the work.

This publication is distributed under The Association of Universities in the Netherlands (VSNU) 'Article $25 \mathrm{fa}$ implementation' project. In this project research outputs of researchers employed by Dutch Universities that comply with the legal requirements of Article $25 \mathrm{fa}$ of the Dutch Copyright Act are distributed online and free of cost or other barriers in institutional repositories. Research outputs are distributed six months after their first online publication in the original published version and with proper attribution to the source of the original publication.

You are permitted to download and use the publication for personal purposes. All rights remain with the author(s) and / or copyright owner(s) of this work. Any use of the publication or parts of it other than authorised under article $25 \mathrm{fa}$ of the Dutch Copyright act is prohibited. Wageningen University \& Research and the author(s) of this publication shall not be held responsible or liable for any damages resulting from your (re)use of this publication.

For questions regarding the public availability of this article please contact openscience.library@wur.nl 


\title{
Environmental Control of Amyloid Polymorphism by Modulation of Hydrodynamic Stress
}

\author{
Jiangtao Zhou, Leonardo Venturelli, ${ }^{\text {Tl }}$ Ludovic Keiser, ${ }^{\text {,I }}$ Sergey K. Sekatskii, François Gallaire, \\ Sandor Kasas, Giovanni Longo, Tuomas P. J. Knowles, Francesco S. Ruggeri,* and Giovanni Dietler*
}

Cite This: ACS Nano 2021, 15, 944-953

ABSTRACT: The phenomenon of amyloid polymorphism is a key feature of protein aggregation. Unravelling this phenomenon is of great significance for understanding the underlying molecular mechanisms associated with neurodegenerative diseases and for the development of amyloid-based functional biomaterials. However, the understanding of the molecular origins and the physicochemical factors modulating amyloid polymorphs remains challenging. Herein, we demonstrate an association between amyloid polymorphism and environmental stress in solution, induced by an air/water interface in motion. Our results reveal that low-stress environments produce heterogeneous amyloid polymorphs, including twisted, helical, and rod-like fibrils, whereas high-stress conditions generate only homogeneous rod-like fibrils. Moreover, high environmental stress converts twisted fibrils into rod-

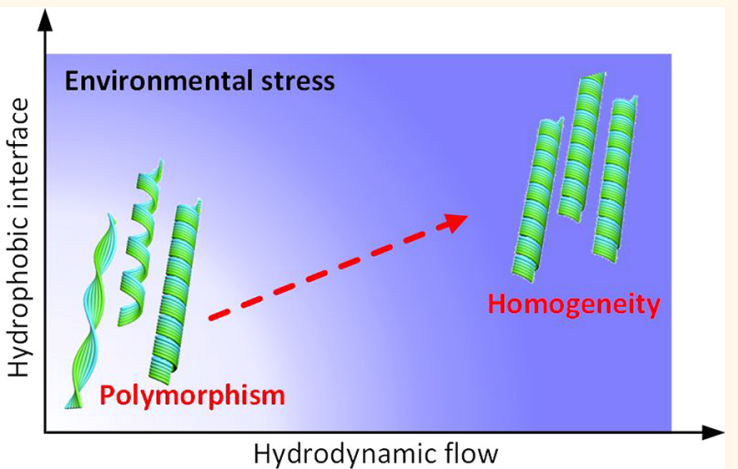
like fibrils both in-pathway and after the completion of mature amyloid formation. These results enrich our understanding of the environmental origin of polymorphism of pathological amyloids and shed light on the potential of environmentally controlled fabrication of homogeneous amyloid biomaterials for biotechnological applications.

KEYWORDS: protein aggregation, amyloid polymorphism, environmental control, air-water interface, hydrodynamic flow

$\mathrm{P}$ athological amyloid formation is a significant feature of many neurodegenerative disorders, such Parkinson's disease, that is related to the misfolding and aggregation of the $\alpha$-synuclein protein. ${ }^{1-4}$ However, amyloids are not only related to disease, and biocompatible functional amyloid materials with exceptional mechanical properties have been gaining increasing interest in biomedical and biotechnological applications, such as biosensors and biomimetic materials. ${ }^{5-7}$

Amyloid polymorphism refers to the diversity of amyloid fibrils' architecture and is a widespread phenomenon in vitro and in vivo. ${ }^{8-12}$ Understanding the underlying mechanisms and pathways of polymorphic amyloid formation is of great importance for both biological and materials science applications. On one hand, various polymorphs of pathologyrelated amyloid fibrils in vivo ${ }^{10,13}$ show distinct biological effects in living systems, e.g., prion strain and cytotoxicity. ${ }^{12,14}$ On the other hand, the performances of functional amyloid materials strongly depend on homogeneity, mesoscale structure, and the deriving mechanical properties, ${ }^{9,11,15-17}$ and the control of amyloid polymorphism could contribute to the development of functional biomaterials. ${ }^{18-21}$
Environmental conditions in the incubation solution are responsible for the modulation of the properties of many biological systems and amyloid formation. ${ }^{22-24}$ Various intrinsic and extrinsic environmental factors have shown significant influence on the process of fibrillar polymorphism ${ }^{25}$ and their aggregation kinetics. ${ }^{26,27}$ It was previously suggested that the interplay among amyloid polymorphs can be controlled by intrinsic kinetics parameters ${ }^{28}$ and induce amyloid polymorphs. ${ }^{8,9,29}$ Furthermore, extrinsic factors, such as hydrophobic air-water interfaces, ${ }^{30-32}$ have shown to significantly accelerate primary nucleation, ${ }^{30,33,34}$ which may impact the morphology of protein aggregates. ${ }^{25,30,32,35}$ Similarly, the presence of hydrodynamic flows strongly influences aggregation kinetics by promoting secondary

Received: September 8, 2020

Accepted: December 17, 2020

Published: December 22, 2020 
a

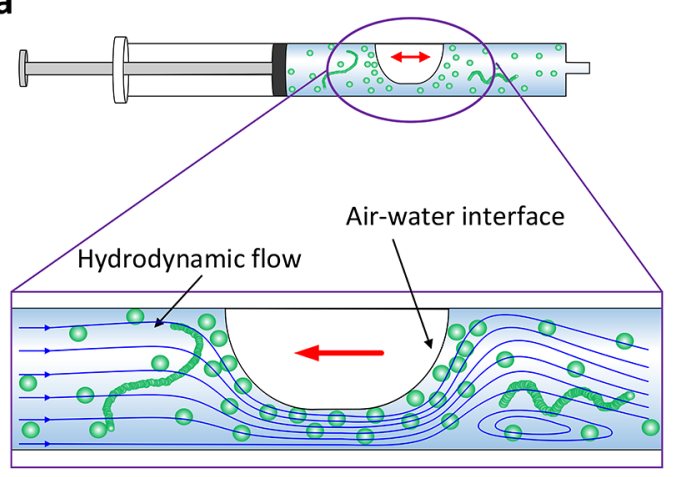

b
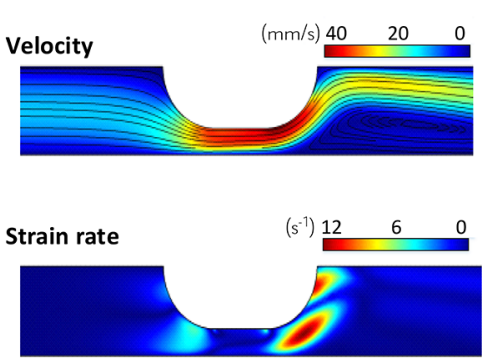

C

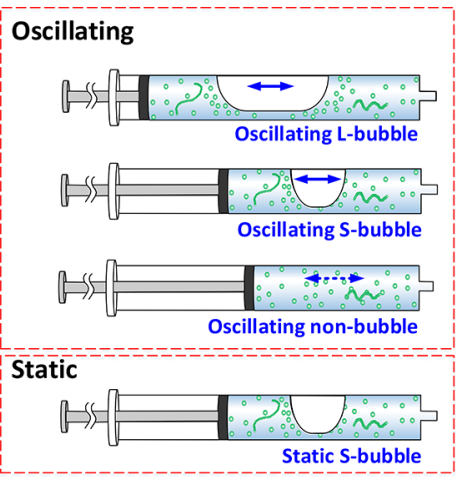

d
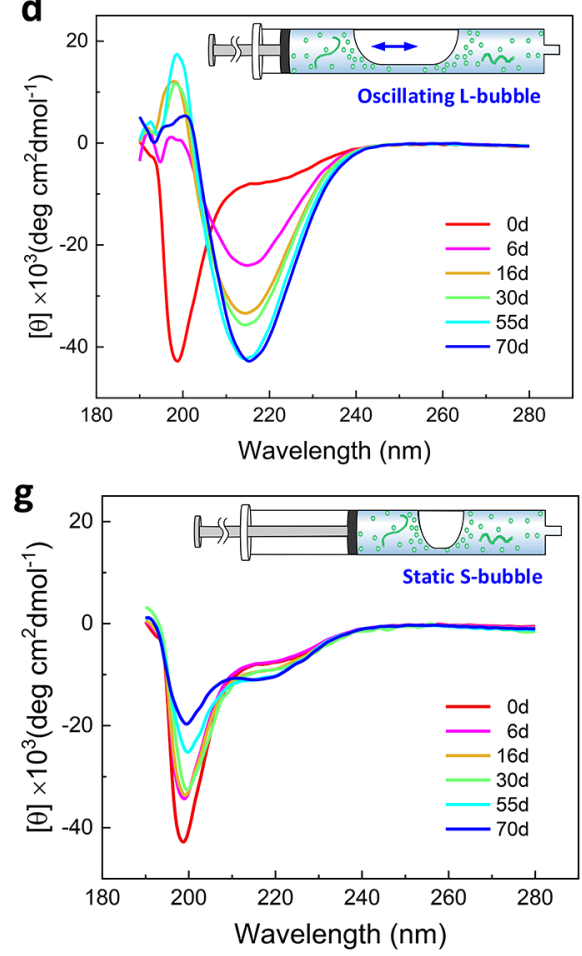

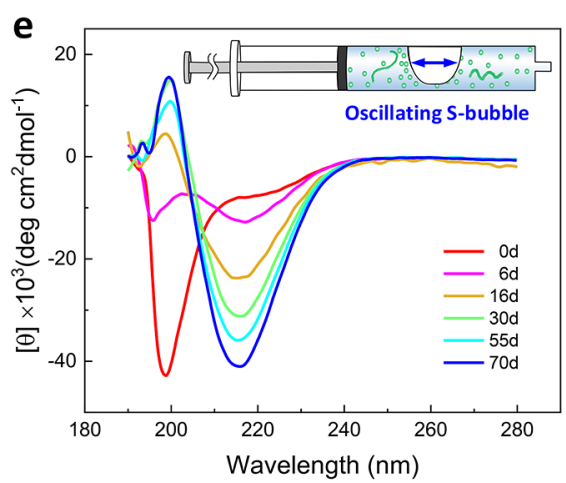

h

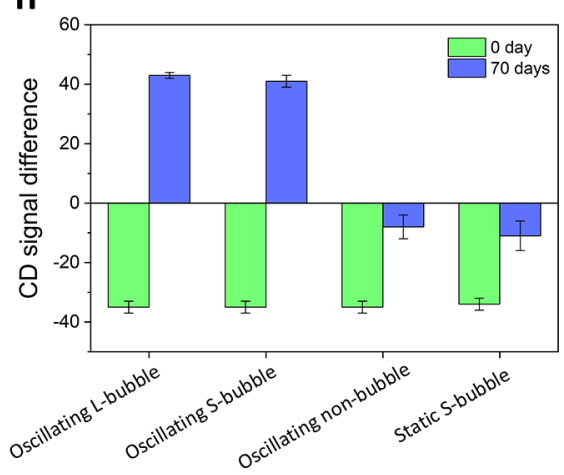

f

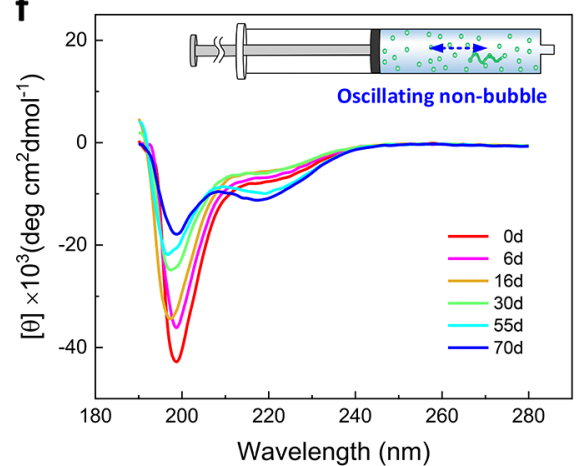

i

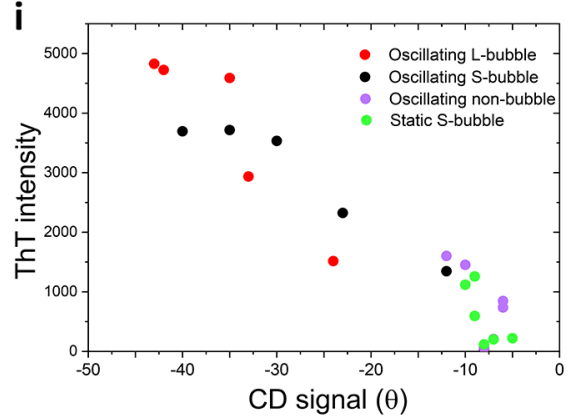

Figure 1. (a) Schematics of the oscillating bubble in the syringe of protein solution exposed to both hydrodynamic and interfacial effects. (b) CFD simulation of oscillating S-bubble condition showing the hydrodynamic flow field and strain rate distribution. (c) Schematics of experimental syringes in the oscillating and static conditions. $(\mathrm{d}-\mathrm{g})$ Evolution of CD spectra under the oscillating L-bubble (d), oscillating S-bubble (e), oscillating nonbubble (f), and static S-bubble (g) conditions. (h) Difference in CD signal between $\alpha$-helix and $\beta$-sheet content after 0 and 70 days of incubation. Error bar represent standard error. (i) Correlation of the CD spectroscopy and ThT fluorescence to characterize the formation of amyloid fibrils.

nucleation $^{33,34,36}$ and protein-protein interaction, ${ }^{26,36-38}$ suggesting a further influence on fibrillar polymorphism. Hydrodynamic and interfacial effects often show a synergetic impact in many physiological circumstances, ${ }^{33,39,40}$ such as bloodstream to cerebrovascular system. ${ }^{41,42}$ Thus, it is of fundamental importance to understand how these extrinsic environmental conditions affect the polymorphism of the aggregates. However, the influence of environmental factors on amyloid polymorphism is still not clear, and the precise modulation of their biophysical properties is still a fundamental challenge in bioscience.

In this work, we achieve the control of the polymorphism of $\alpha$-synuclein $(\alpha$-Syn) amyloid fibrils by modulation of the degree of hydrodynamic stress in solution. This approach is realized by regulating the size of an air-water interface introduced in the incubation tube in the form of an air bubble and the strength of hydrodynamic flow induced by rotation of the tube in a microgravity random positioning machine. We then pursue a single aggregate structural characterization ${ }^{43-45}$ of polymorphism as a function of the variation of hydrodynamic stress in solution and demonstrate that the control of amyloid polymorphism can be achieved on-pathway or after the formation of mature amyloid fibrils. These results contribute to the understanding of the origin of pathological amyloid polymorphism, as well as shed light on controlling fibrillar polymorphism, in order to produce homogeneous amyloid-based functional biomaterials.

\section{RESULTS AND DISCUSSION}

Control of Environmental Hydrodynamic Stress during $\boldsymbol{\alpha}$-Synuclein Aggregation. The study of $\alpha$-Syn amyloid formation was carried out by controlling the presence 

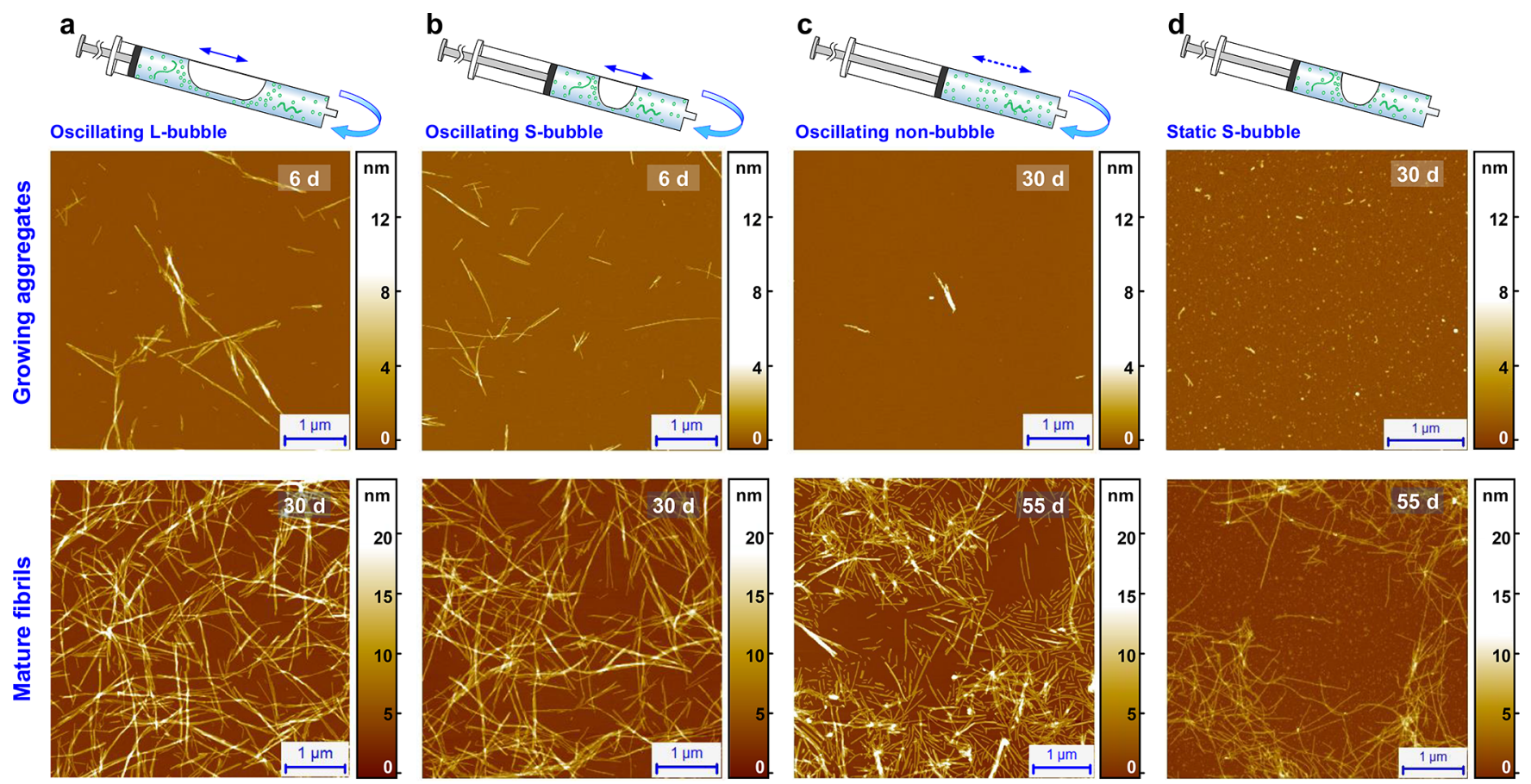

e

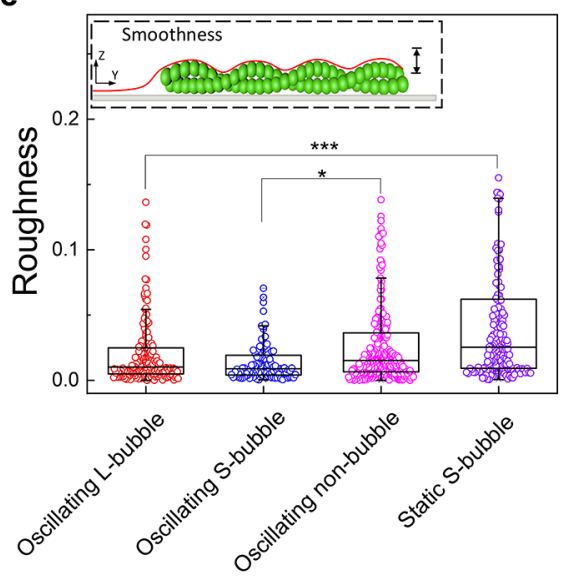

f

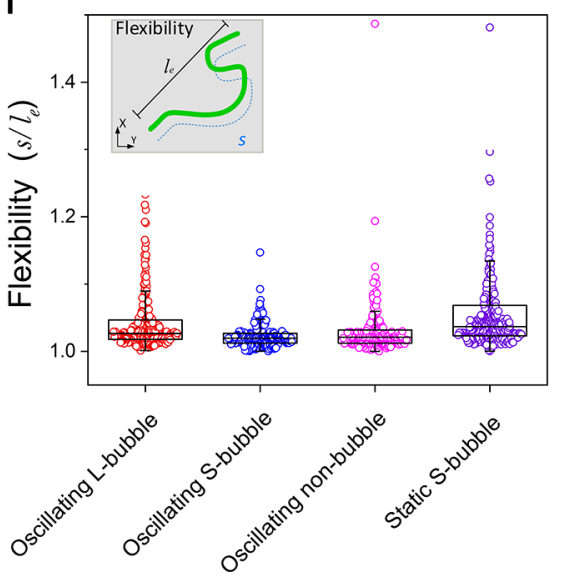

g

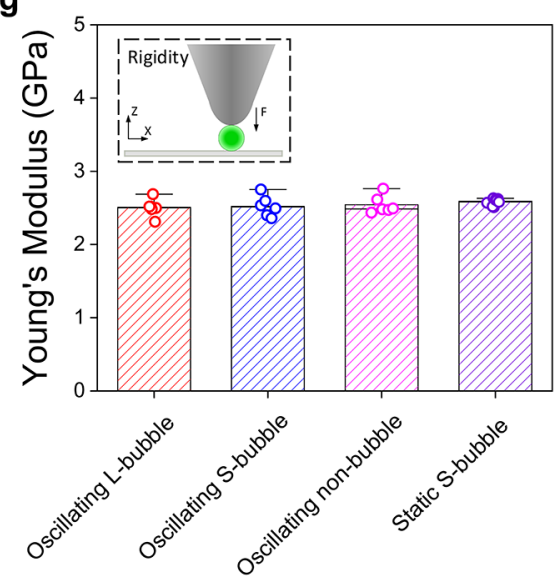

Figure 2. AFM images and statistical analysis on the representative features of amyloid fibrils. (a-d) AFM images of growing aggregates and mature fibrils from each studied condition at the concentration of $30 \mu \mathrm{M}$. (e-g) Statistical investigation on the morphological and mechanical properties of more than $\mathbf{1 5 0}$ mature fibrils in each condition, including the roughness (e), flexibility (f), and Young's modulus (g). Statistically significant differences are noted $(*: p<0.05, * * *: p<0.001)$ that were determined by using two-way ANOVA with Tukey's posthoc correction. Error bar, SD.

and the motion of an air bubble introduced in the protein solution incubated in a syringe rotating in a random positioning machine (RPM) (Figure 1a,b). ${ }^{46-48}$ The bubble introduces an air-water interface, and the rotation at a low speed allows a gentle reciprocating motion of the bubble in the syringe, which further induces a hydrodynamic flow in solution (see Methods and Figure S1). The protein was incubated at the concentration of $30 \mu \mathrm{M}$.

To study the effect of the hydrodynamic flow and air-water interface systematically, three syringes of $\alpha$-Syn solution were prepared with different bubble sizes: large bubble (oscillating L-bubble), small bubble (oscillating S-bubble), and no bubble (oscillating nonbubble), as shown in Figure 1c. The volume ratios of protein solution to bubble in the large and small bubble conditions were maintained 2:1 and 4:1, respectively, in the experiment (see Methods). Since both the hydrodynamic flow and hydrophobic air-water interface could influence the kinetics of $\alpha$-Syn fibril formation, ${ }^{34,35}$ we also studied as control the aggregation of $\alpha$-Syn in another syringe with a small bubble and incubated in the static condition (static Sbubble) to discriminate the effect of the air-water interface alone.

In the case of the oscillating L/S-bubble conditions, the gentle bubble movement (Figure 1a) led to a combined environmental stress, originated from the air-water interface and the generated hydrodynamic flow. To quantify the strength of these hydrodynamic and surface effects, we performed a computational fluid dynamics (CFD) simulation (Figures $1 \mathrm{~b}$ and S2). The simulation indicated the main hydrodynamic contribution, the strain rate (Methods), which refers to the elongation flow fields. ${ }^{36}$ The simulation showed that the hydrodynamic flow is as large as $\sim 45 \mathrm{~mm} / \mathrm{s}$ and 
generates high kinetics stress with a strain rate of $\sim 12 s^{-1}$, in both oscillating L-bubble and oscillating S-bubble conditions. No significant difference of flow field and strain rate values was observed between these two conditions (Figure S2). Therefore, the main difference between the oscillating L-bubble and oscillating S-bubble conditions was the different surface to volume ratio of the air-water interface. The volume of the air bubble in the former case was maintained at twice larger than that of the latter case in this experiment. We then refer to these two conditions as high hydrodynamic stress environments.

By contrast, the protein solution in the oscillating conditions in the absence of any air-water interface (oscillating nonbubble) was affected only by the residual hydrodynamic stress due to the agitation of dissolved microbubbles, which are always present in solution, ${ }^{49,50}$ and the insoluble protein aggregates forming as a function of time. Similarly, the protein solution in the static condition (static S-bubble) was only exposed to an air-water interface in the absence of any hydrodynamic effect. We therefore refer to these two conditions as low hydrodynamic stress environments.

Bulk Characterization of $\alpha$-Syn Amyloid Fibril Formation. The structural evolution of $\alpha$-Syn aggregates was investigated by circular dichroism (CD). This technique enables monitoring the secondary structure conformation of protein during amyloid formation. Prior to incubation, $\alpha$-Syn showed a characteristic absorption at $199 \mathrm{~nm}$ (Figure $1 \mathrm{~d}-\mathrm{g}$ ), indicating an intrinsic disordered random-coil structure that is characteristic of monomeric proteins. In all conditions, as a function of time, this peak gradually shifted to a minimum at $218 \mathrm{~nm}$, demonstrating a conversion from random coil to $\beta$ sheet conformation and indicating the formation of cross $\beta$ sheet amyloid fibrils.

In the oscillating L-bubble condition (Figure 1d), already after 6 days, the absorption dip at $199 \mathrm{~nm}$ disappeared and the dip at $218 \mathrm{~nm}$ increased in the spectrum. The structural transition in oscillating S-bubble (Figure 1e) condition showed a slightly slower but similar trend. By contrast, the spectral variations in the oscillating nonbubble (Figure 1f) and static Sbubble (Figure $1 \mathrm{~g}$ ) conditions occurred at similar and much slower speed. During 70 days of incubation, a gradual decrease of the dip at $199 \mathrm{~nm}$ and a slight rise of the dip at $218 \mathrm{~nm}$ were simultaneously observed on the spectra. Furthermore, the smaller amplitude of the peak at $218 \mathrm{~nm}$ in these two conditions, after 70 days of incubation, suggested the formation of a lower quantity of amyloid fibrils when compared to the oscillating L/S-bubble conditions at the same time point of aggregation.

The CD results showed a significant formation of $\beta$-sheet content in the high hydrodynamic stress environment of the oscillating L/S-bubble conditions (Figure $1 \mathrm{~h}$ ), whereas this conversion was less extensive under low environmental stress conditions, i.e., under RPM oscillation of the solution or in the presence of a static air-water interface. These results were confirmed by a thioflavin $\mathrm{T}$ (ThT) fluorescence assay (Figure S3 and Supplementary Text 1). The ThT signals reached the final plateau of the aggregation reaction after 25 days, in the oscillating L/S-bubble conditions, while the plateau was reached after more than 50 days in the oscillating nonbubble and static S-bubble conditions. We then plotted the intensity of ThT fluorescence versus the CD intensity at $218 \mathrm{~nm}$ (Figure 1i) to confirm the tendency of protein aggregate formation in both conditions of high and low hydrodynamic environmental stress. $^{34}$
Single Aggregate Characterization of $\alpha$-Syn Amyloid

Formation. The morphological differences of amyloid aggregates play a critical role in determining various biological activities, e.g., cytotoxicity, ${ }^{12,14}$ and biomaterial performance such as mechanical properties. ${ }^{18-21}$ After the bulk characterization, we then employed high-resolution atomic force microscopy (AFM) to assess morphological features of amyloid fibrils at the single-aggregate level.

We first imaged the process of amyloid formation as a function of the incubation time (Figure $2 a-d$ ), from the initiating monomeric protein (Figure S4). Then, we characterized the morphological characteristics of the formed fibrils. We evaluated the length and average height of the fibrils, as well as their smoothness, flexibility, and stiffness (Figure $2 \mathrm{e}-\mathrm{g}$ and Figures S5-7). The smoothness described the surface roughness of the fibrils ${ }^{21,51}$ (Figure S5). The flexibility, characterized by the ratio of contour length $s$ to end-to-end length $l_{\mathrm{e}}\left(s / l_{\mathrm{e}}\right.$, Figure $\left.\mathrm{S} 8\right)$, is related to the fibril stiffness and to its interstrand cross-sectional arrangement. ${ }^{44,52-54} \mathrm{~A}$ lower value of flexibility is related to an increase of the bending rigidity, which is correlated to the mechanical properties of the aggregates. To correlate the flexibility to the Young's modulus of mature fibrils, we thus performed AFM quantitative nanomechanical mapping of Young's modulus ${ }^{20}$ (Figure $2 \mathrm{~g}$, Figure S9 and Supplementary Text 2).

Morphological and kinetics differences in the aggregation of the protein under the conditions investigated (high and low hydrodynamic environmental stress) became gradually evident as a function of the incubation time, as shown in Figure $2 \mathrm{a}-\mathrm{d}$. Prior to incubation, $\alpha$-Syn protein was in the monomeric and oligomeric state (Figure S4). In the oscillating L-bubble condition (Figure $2 \mathrm{a}$ and red boxes in Figure $2 \mathrm{e}-\mathrm{g}$ ), protofibrils and fibrils formed only after 6 days, while abundant mature fibrils were obtained after 30 days of incubation. Similarly, the oscillating S-bubble conditions (Figure $2 \mathrm{~b}$ and blue boxes in Figure $2 \mathrm{e}-\mathrm{g}$ ) promoted fibril formation, and abundant mature fibrils were detected after 30 days of incubation. Interestingly, the morphological features of mature fibrils yielded in the two high-stress conditions are comparable, with similar fibril average length and diameter (Figure S6). In both conditions, the fibrils showed relatively low flexibility and low roughness (Figure 2e,f).

In the oscillating nonbubble condition (Figure $2 \mathrm{c}$ and magenta boxes in Figure $2 \mathrm{e}-\mathrm{g}$ ), the trend of amyloid fibril formation was slower. Protofibrillar and fibrillar aggregates were detected after 30 days, and short mature fibrils (average length $0.2 \pm 0.1 \mu \mathrm{m}$ ) were formed after only 55 days of incubation. We could demonstrate that these are mature fibrils since they possess high roughness and rigidity (Figure 2e,f), with a relatively uniform average height of $5 \pm 0.7 \mathrm{~nm}$ (Figure S6).

By contrast, the slower static S-bubble condition (Figure $2 \mathrm{~d}$ and purple boxes in Figure $2 \mathrm{e}-\mathrm{g}$ ) showed the abundant formation of only protofibrillar aggregates (height of peaking $3.1 \pm 0.2 \mathrm{~nm}$ ) after 30 days, $^{30,34}$ and only after 55 days we had the formation of an abundant population of mature amyloid fibrils with an average height of $5.2 \pm 0.4 \mathrm{~nm}$ (Figures S6 and S7).

Overall, the protein solutions under high environmental stress showed the abundant formation of amyloid fibrils after 6 days of incubation, while the fibrils under low environmental stress formed amyloid fibrils only after 50 days of incubation. 

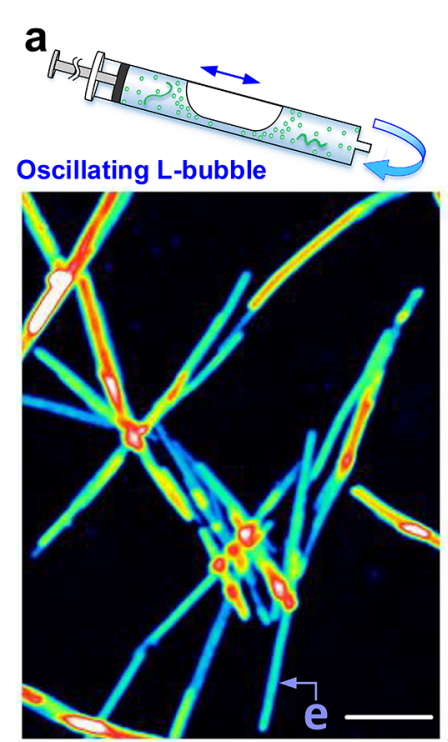

b
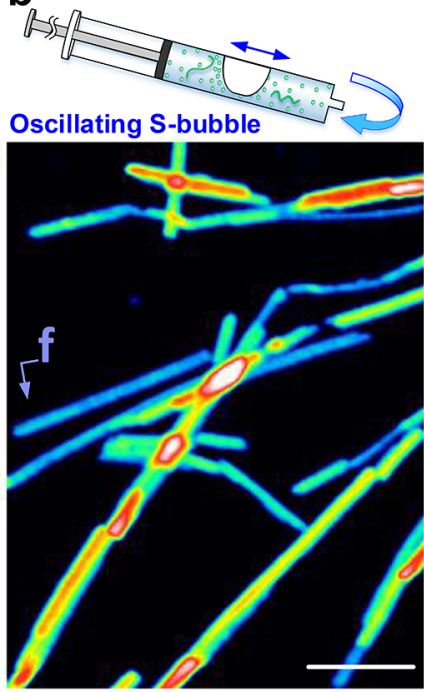
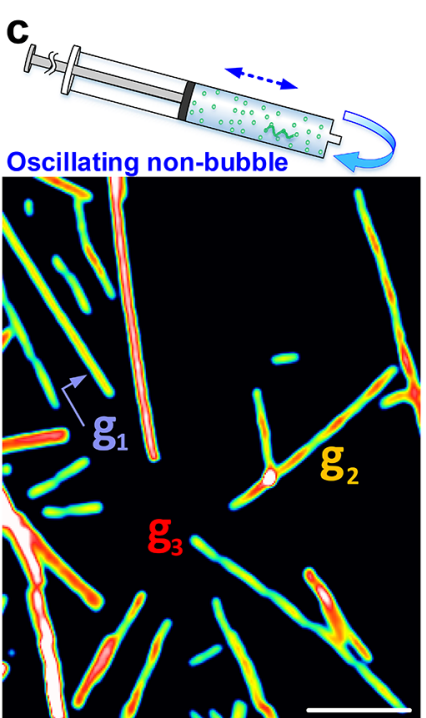
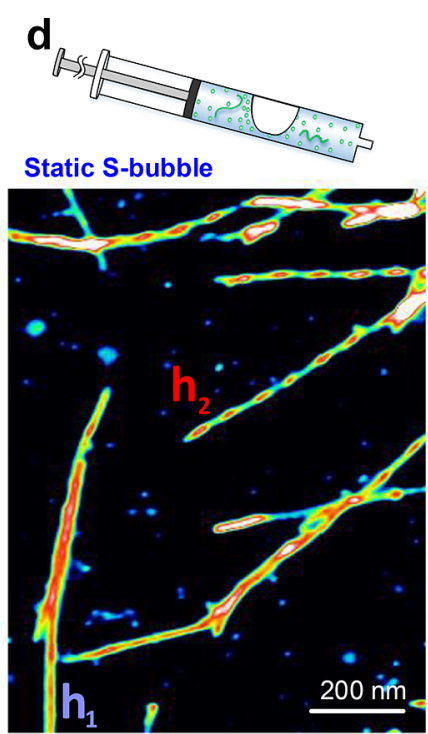

e

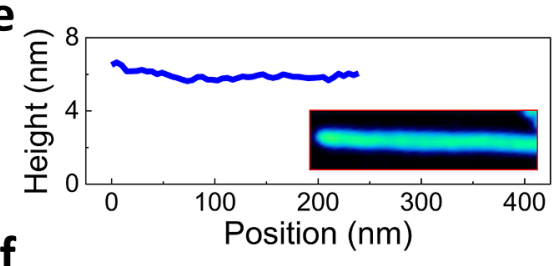

f

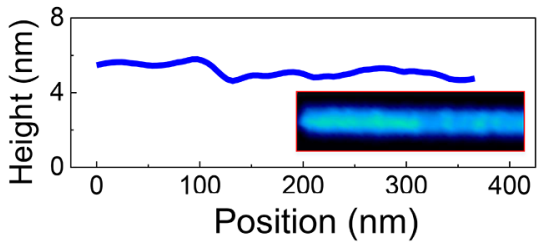

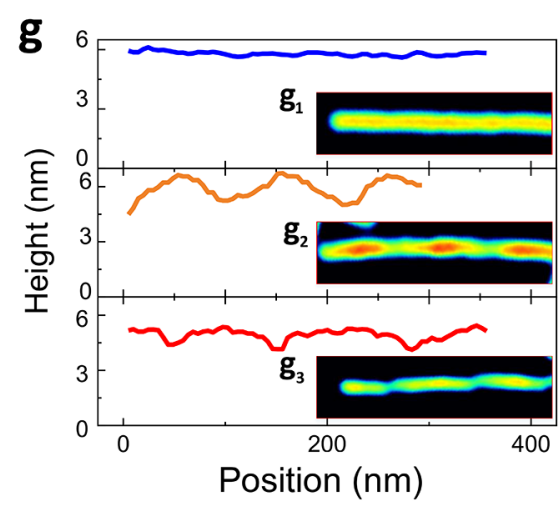

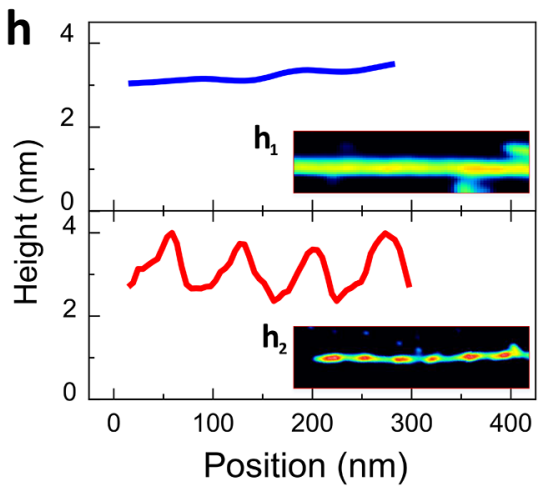

i

Oscillating L-bubble

Oscillating S-bubble

Oscillating non-bubble

Static S-bubble
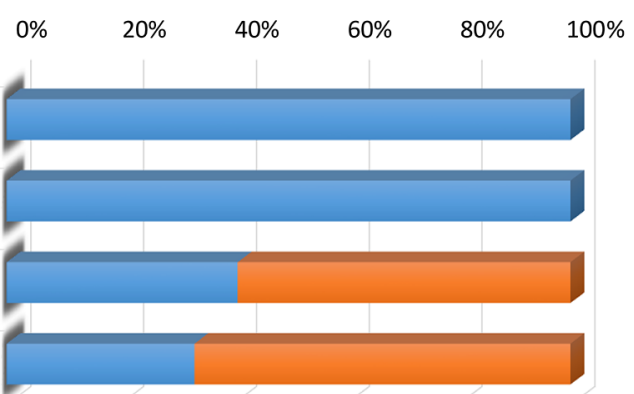

- Rod-like - Twisted \& Helical

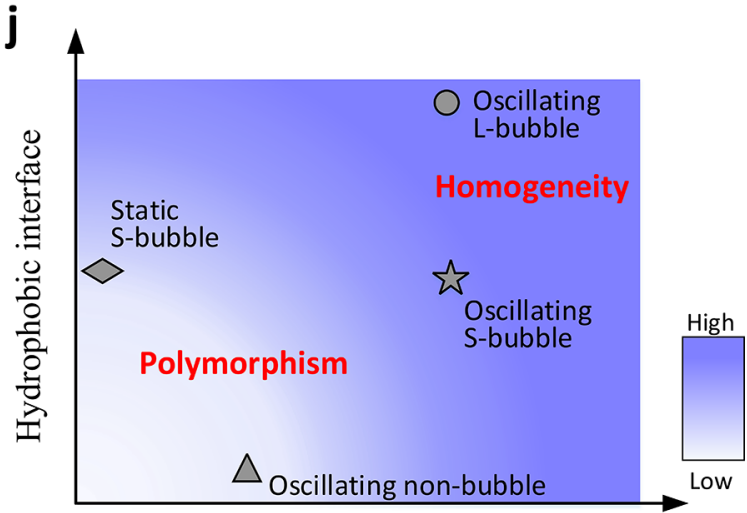

Hydrodynamic flow

Figure 3. Fibril polymorphism and its correlation with environmental stress. (a-d) AFM images of fibrils formed. In the oscillating L-bubble (a) and oscillating L-bubble (b) conditions, we observed only rod-like fibrils (e, f). In the oscillating nonbubble (c) and static S-bubble (d) conditions, we observed a mixture of twisted, helical, and rod-like polymorphs $(\mathrm{g}, \mathrm{h})$. (i) Statistics of amyloid polymorphism in each condition from more than $\mathbf{1 0 0}$ mature fibrils. (j) Plot of environmental stress of each incubation condition originated from hydrodynamic flow and air-water interface to indicate the dependence between fibril polymorphism and environmental stress. The strength of the blue color indicates the strength of the environmental stress, where the deep-blue zone produces homogeneous fibrils and the light-blue zone generates polymorphic fibrils.

\section{Single-Aggregate Characterization of $\alpha$-Syn Amyloid} Polymorphism. As a major advantage of AFM over other imaging methodologies, we performed nanoindentation measurements to prove that we were comparing only the morphology of mature amyloid fibrils. The measured Young's modulus was $\sim 2.5 \mathrm{GPa}$ (Figures $2 \mathrm{~g}$ and S9), as expected for mature amyloid fibrils and supported by the ThT assay (Figure S2). Being sure to compare the structural state of amyloid fibrils at the same final stage of amyloid formation, we performed the statistical investigation of their polymorphism 
a
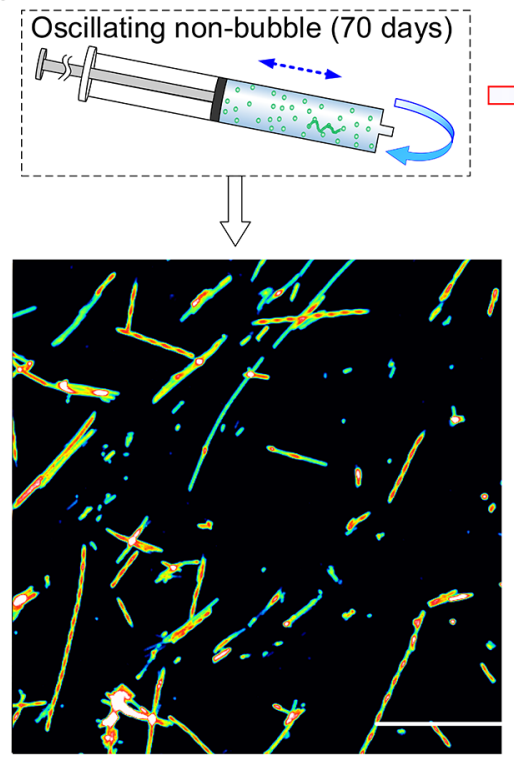

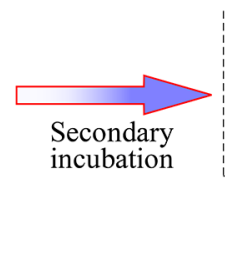

Oscillating S-bubble (5 days)
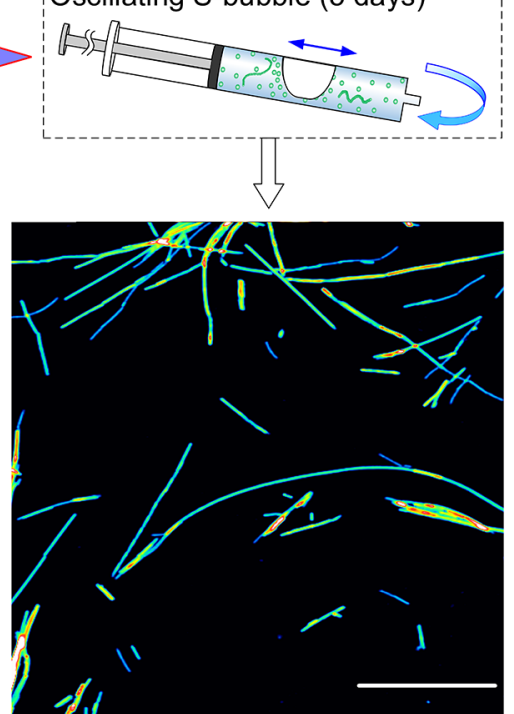

b

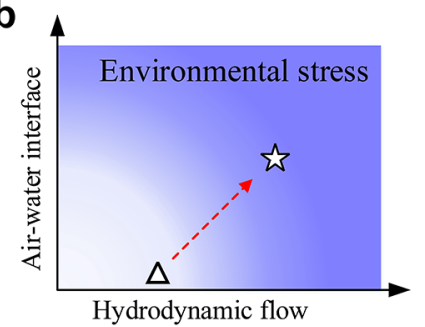

C

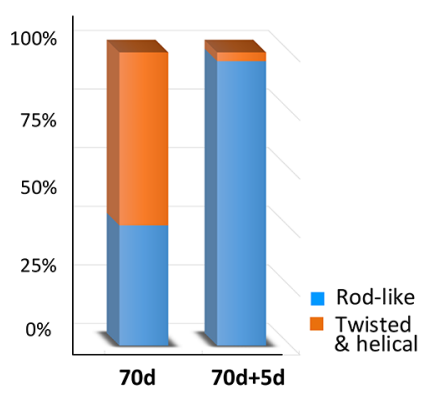

d

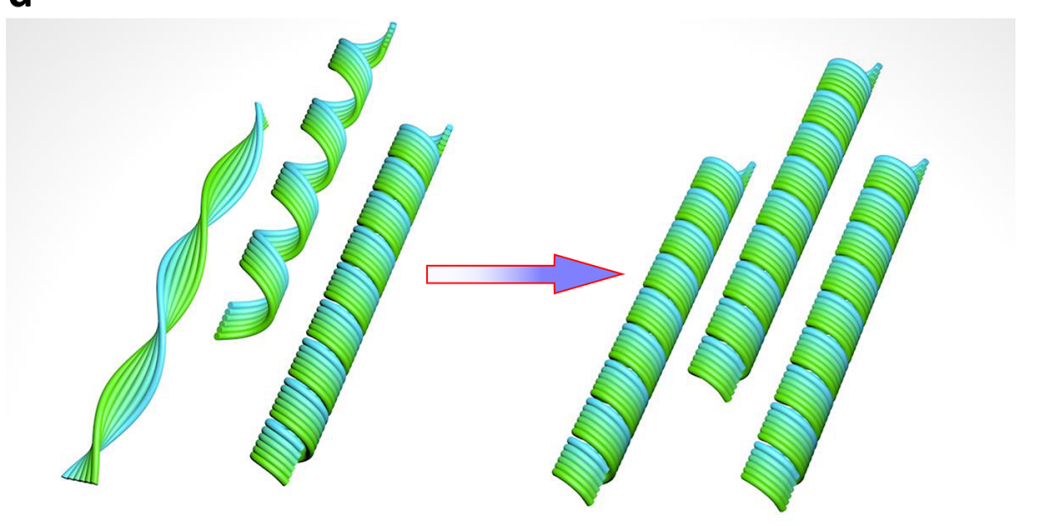

e

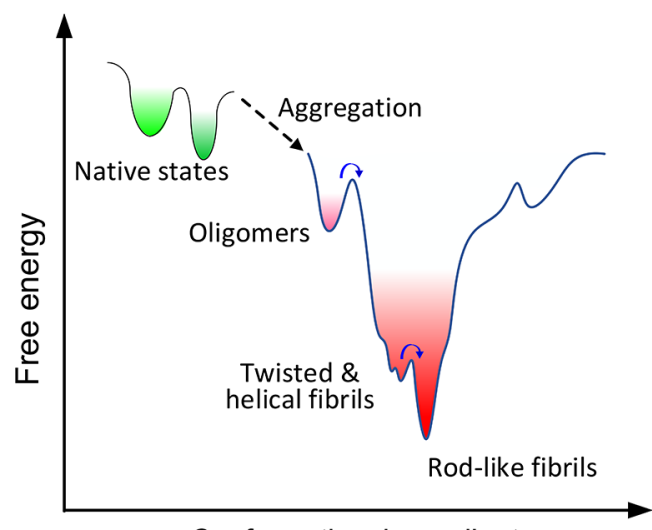

Conformational coordinate

Figure 4. Order-order transition of amyloid polymorphs by mediating environmental stress. (a) Protein solution incubated after 70 days in the oscillating nonbubble condition yielded mature fibrils with heterogeneous morphologies (left). After the secondary incubation of 5 days in the oscillating S-bubble condition, these fibrils transformed into rod-like fibrils (right). (b, c) Variation of the environmental stress (b) and the statistics of fibril polymorphs (c) before and after the transition based on the observation of more than 150 amyloid fibrils. (d) Schematics of the transition from polymorphic fibrils to homogeneous rod-like fibrils. (e) Funnel-like energy landscape map of the amyloid polymorphs in this experiment.

(Figure $3 \mathrm{a}-\mathrm{d}$ ). The oscillating L-bubble and oscillating Sbubble conditions promoted the formation of homogeneous rod-like fibrils (Figures $3 \mathrm{a}, \mathrm{b}$ and $\mathrm{e}, \mathrm{f}$ ), which indeed had very low surface roughness (Figure 2e). Multiple fibril polymorphs were found in the oscillating nonbubble and static S-bubble conditions, including rod-like, ${ }^{8,16,12,18}$ helical, ${ }^{8,9,16,18}$ and twisted $^{8,15,16,18}$ fibrils (Figure $3 \mathrm{c}, \mathrm{d}$ and g,h). Thus, the incubation conditions with high environmental stress generated homogeneous rod-like fibrils, whereas the conditions with weak stress could lead to polymorphic fibrils. This result is supported by the previous observations on the fibril formation influenced by hydrodynamic flow ${ }^{55}$ or interfacial factor ${ }^{35,56}$ individually, as well as their synergistic effect. ${ }^{57}$

The different degree of polymorphism in the different conditions is related to the environmental stress, which can thus be used to control the polymorphism of amyloid fibrils. ${ }^{11,17,28,58}$ In this designed experiment, the environmental stress in the oscillating nonbubble and the static S-bubble condition were relatively weak, as shown on the environmental stress map (Figure 3j). This weak stress allows multiple fibrillization pathways and thus yields heterogeneous fibrils, including twisted, helical, and rod-like polymorphs, while the high environmental stress in the oscillating L-bubble and oscillating S-bubble conditions leads to the homogeneous formation of the most thermodynamically stable rod-like fibrils.

Controlling and Modulation of Amyloid Polymorphism. To further demonstrate the capability of environmental stress in controlling amyloid polymorphism, we considered the secondary incubation under high environmental stress of mature amyloid fibrils formed under low environmental stress. First, the protein solution was incubated for 70 days in the oscillating nonbubble condition (low environmental stress), which yielded mature twisted and rodlike amyloid fibrils, as confirmed also by the ThT fluorescence 
assay (Figure S3). Then, this solution was further incubated for 5 days in the oscillating S-bubble condition (high environmental stress), as shown in Figure 4a. This approach resulted in changing environmental stress of the final fibrillar products of aggregation from low to high stress (Figure 4b). Prior to the secondary incubation, under low environmental stress, we detected the coexistence of multiple polymorphs including twisted and rod-like fibrils. However, after 5 days in the oscillating S-bubble condition, in high environmental stress, a clear transition was observed from heterogeneous polymorphs to homogeneous rod-like fibrils, as demonstrated by a single aggregate statistical investigation (Figure 4c). Thus, we demonstrated that the amyloid fibril polymorphism could be successfully modulated by varying environmental stress in solution, even after mature fibril formation.

We suggest that lower environmental stress results in the formation of several polymorphs of amyloid fibrils because it could allow a higher number of multiple aggregation pathways compared to the higher environmental stress, which result in the formation of homogeneous and more thermodynamically stable rod-like fibrils (Figure 4d). Furthermore, the correlation between amyloid polymorphism and environmental stress could also be discussed in terms of the energy landscape of protein aggregation. From an energetic point of view, the environmental-stress-dependent transition from twisted and helical fibril polymorphs to rod-like fibrils suggests that amyloid polymorphs in this experiment may hold certain levels of free energy and structural stability, as shown in Figure 4e. The twisted and helical fibrils are the metastable and transformable species that occupy a higher level on the energy landscape and form under low environmental stress. Under high environmental stress, the fibrils are able to transit to rodlike polymorphs that occupy a lower energy level. Thus, in our conditions, the rod-like fibrils are the thermodynamically most stable form with minimum free energy in the energy landscape. $8,18,29,59,60$ In addition, parallel stacked rod-like fibrils, also termed multistranded ribbon, were observed occasionally in the high-environmental-stress conditions after long-term (70 days) incubation (Figure S10 and Supplementary Text 3), which might also maintain a similar or even lower energy level compared to rod-like fibrils., ${ }^{9,59}$

\section{CONCLUSIONS}

In summary, our results demonstrate that amyloid fibril polymorphism can be modulated by the environmental hydrodynamic stress in solution, both in-pathway and after mature amyloid fibril formation. We observed that polymorphic twisted and rod-like amyloid fibrils were formed in low hydrodynamic environmental stress conditions, while homogeneous rod-like fibrils were yielded in the high hydrodynamic environmental stress conditions. The results suggest that twisted, helical, and rod-like fibrils may occupy distinct free-energy levels on the energy landscape. Twisted and helical fibrils are metastable states that transit to the more thermodynamically stable and energetic-favorable rod-like polymorphs with a lower energy level. The environmental control in solution of the order-order transition from heterogeneous (twisted, helical, rod-like polymorphs) to homogeneous (rod-like) fibrils may facilitate the production of homogeneous protein aggregates from heterogeneous amyloid polymorphs. This approach shows great potential for the development of future functional amyloid-template biomaterials, with homogeneous conformation for materials science and biomedical applications.

\section{METHODS AND MATERIALS}

Sample Preparation. Full-length recombinant human $\alpha$ synuclein (AnaSpec, USA) was dissolved in buffer $(50 \mathrm{mM}$ Tris. $\mathrm{HCl}, 150 \mathrm{mM} \mathrm{NaCl}, \mathrm{pH} 7.4)$ and filtered with a $100 \mathrm{kDa}$ filter. The concentration of the $\alpha$-Syn solution was measured by ultraviolet absorbance (Nanodrop 2000). An aliquot $(1 \mathrm{~mL})$ of $\alpha$-Syn solution was sealed in sterilized low protein binding wall syringes and placed in different incubation conditions (Figure S1) at a concentration of 30 $\mu \mathrm{M}$ at $37^{\circ} \mathrm{C}$. Three syringes were incubated in the absence and presence of a large or small bubble in the RPM conditions, and another syringe with a small bubble was statically incubated. In the large (L-bubble) and small bubbles (S-bubble) the ratio of the volume of protein solution was maintained at 2:1 and 4:1, respectively, throughout the experiment. The size of the surface of the air bubble in the L-bubble condition is roughly 2-3 times larger than that in the Sbubble condition.

Rotating Bioreactor System. The three oscillating syringes with different sizes of bubbles were incubated on the platform of an inhouse-developed random positioning machine at $37^{\circ} \mathrm{C}$. The rotation of the platform was regulated by two independent motors in three dimensions, which enables the rotation in random directions. The samples were fixed at the center of the RPM, and a rotation speed of RPM of $\sim 5 \mathrm{rpm}$ was used to reduce the gravity effects of sedimentation and to maintain a gentle bubble oscillation in the syringe.

CFD Simulation. To quantify the hydrodynamics generated by bubble motion, we performed a CFD simulation using the finite elements method. The oscillating bubble generates a hydrodynamic flow in the capillary, and its flow field is mainly contributed by the strain rate (Figure S2), referring to the extensional flow field. This strain rate (elongational) is known to be crucial for protein aggregation due to its ability to stretch protein molecules and to increase protein kinetics compared with the shear rate. ${ }^{36}$ In Figure $1 \mathrm{~b}$, the strain rate maximized, on the order of $10 \mathrm{~s}^{-1}$, at the rear and at the front side of the bubble, due to an intermediate Reynolds number (Re $=60$ ) of the protein solution. Meanwhile, no significant differences of flow field and strain rate values were observed between the oscillating L-bubble and the oscillating S-bubble conditions (Figure S2).

The CFD simulations were carried out by using the laminar flow module of the finite element method (FEM) solver Comsol (COMSOL Inc., MA, USA). To account for the flow created by the buoyancy-driven bubble oscillation in the experiments, we performed the simulation in the frame of the bubble. The dimensions of the capillary were $1 \times 16 \mathrm{~cm}$, while the height of the bubble was 7 $\mathrm{mm}$. The no-slip boundary condition was applied to the walls of the capillary, and the interface of this air bubble was considered to be shear-free (full slip) as a reasonable assumption for air-liquid interfaces. A flow of constant flow rate $q=V d$ was injected from the left of the capillary, where $d$ is the capillary width and $V$ is the mean velocity of the flow. Here we took $V=10 \mathrm{~mm} / \mathrm{s}$ to meet the velocity of bubble motion in the experiment, which corresponds to a Reynolds number:

$$
\operatorname{Re}=\rho V d / \mu=60
$$

where $\rho$ and $\mu$ are the density and viscosity of the protein solution, respectively. We neglected the orientation variation of the oscillatory flow, as the hydrodynamic flow can be represented in each cycle and the strain rate distributes mainly due to the expansion of the flow independent from bubble oscillation. Navier-Stokes equations were solved in the bidimensional domain composed of 18433 mesh elements and 801 boundary elements (corresponding to 28854 degrees of freedom solved). The simulations converged, and doubling the number of mesh elements led to the same results. The strain rate $(s t)$ is defined in the following as 


$$
s t=\sqrt{\left(\frac{\partial u}{\partial x}\right)^{2}+\left(\frac{\partial v}{\partial y}\right)^{2}}
$$

where $u$ and $v$ respectively represent the velocity field component in the $x$ and $y$ direction. The strain rate is characterized by the norm of the gradient of the flow velocity in the direction parallel to the streamline that corresponds to the extensional flow field. This choice for the formula provided a close estimation of the force to which protein oligomers and aggregates are subjected.

CD Spectroscopy. An aliquot of diluted $\alpha$-Syn solution $(15 \mu \mathrm{M})$ was analyzed at room temperature with a Jasco J-815 CD spectrometer in the range of $190-280 \mathrm{~nm}$ in each measurement. A high-quality quartz cuvette with an optical path length of $1.0 \mathrm{~mm}$ was employed, and spectra were collected with a resolution of $0.2 \mathrm{~nm}$ in continuous scanning mode. Further smoothing of spectra was processed with a Savitzky-Solay filter in OriginPro (OriginLab, MA, USA).

ThT Assay. Fresh ThT solution was prepared from a stock solution before each measurement at the ThT concentration of 100 $\mu \mathrm{M}$. An aliquot of $\alpha$-Syn solution was diluted by a ThT solution and Milli-Q water to reach a final protein concentration of $3 \mu \mathrm{M}$ and ThT concentration of $10 \mu \mathrm{M}$ in every experiment. An aliquot of $\alpha$-Syn solution $(70 \mu \mathrm{L})$ in each experiment was measured three times in a Bucher Analyst $\mathrm{AD}$ plate reader. The ThT fluorescence reading was performed at an excitation wavelength of $450 \mathrm{~nm}$ and an emission wavelength of $485 \mathrm{~nm}$. The signal of fluorescence intensity was fitted with a sigmoidal model according to

$$
y(t)=\frac{A}{1+\mathrm{e}^{-\left(t-t_{50}\right) / \tau}}
$$

where $A$ is the difference in fluorescence intensity between the plateau and baseline on the ThT curve, $t$ is the incubation time, $t_{50}$ is the time at which the signal reaches half of the average value of the plateau and baseline, and $1 / \tau$ is the rate of fibril growth. ${ }^{26,30,45}$

AFM Measurement. Aliquots of $\alpha$-Syn solution $(30 \mu \mathrm{M})$ were collected at each time point and diluted to $10 \mu \mathrm{M}$ before each AFM measurement. Freshly cleaved mica was functionalized by an aliquot $(10 \mu \mathrm{L})$ of freshly prepared $1 \%$ (3-aminopropyl)triethoxysilane for $1.5 \mathrm{~min}$ and then rinsed with Milli-Q water and dried using compressed air flux. Then, an aliquot $(10 \mu \mathrm{L})$ of $\alpha$-Syn solution was deposited on the mica for $4 \mathrm{~min}$, followed by a smooth rinsing of Milli-Q water and a gentle flow of nitrogen gas.

AFM measurements were operated by a Park NX10 AFM (Park Systems, South Korea) in noncontact mode using NCHR cantilevers (Nanosensors, Switzerland), with a nominal spring constant of $42 \mathrm{~N} /$ $\mathrm{m}$ and a typical tip radius of less than $7 \mathrm{~nm}$. A soft tip-sample interaction was applied during scanning by monitoring the phase of the tapping cantilever at less than $\pm 5^{\circ}$ to ensure a consistent, accurate AFM measurement. ${ }^{21}$ AFM images were simply flattened using XEI software (Park Systems, South Korea) by first- and second-order line subtraction.

AFM Statistical Analysis. The statistical analysis of flattened AFM images was carried out using the DNA-trace software, previously home-developed. ${ }^{61}$ The height profile of mature amyloid fibrils was traced along the ridge of fibrils by selecting the maximal height point in every cross-section. The statistical determination of fibril length was obtained by measuring the fibril contour length, and importantly, the average height and smoothness were recorded by tracing fibrils while avoiding the overlapped fibril regions (Figure S5). The average height was calculated by averaging the height profile along the fibril, and smoothness was measured by counting $80 \%$ variation of the height profile of the amyloid fibril.

QI-AFM Imaging. QI-AFM imaging was performed by using a JPK Nanowizard III microscope (Bruker, USA) with a cantilever (PPP-CONTSCAuD, Nanosensors) with a nominal spring constant of $0.2 \mathrm{~N} / \mathrm{m}$ and a typical tip radius of less than $7 \mathrm{~nm}$. The spring constant and deflection sensitivity of the cantilever were calibrated before each measurement. All QI-AFM images are acquired in the quantitative imaging (QI) mode with a size of $5 \times 5 \mu \mathrm{m}^{2}$ and a resolution of $256 \times 256$ pixels, and each pixel represents a forcedistance (FD) curve recorded within $20 \mathrm{~ms}$. At least five QI-AFM images were collected at different locations of each sample. Image processing was processed using JPK Data Processing software (Bruker, USA) with the following steps: calibration of the spring constant and deflection sensitivity; correction of the baseline offset and tilt; determination of the contact point; and elasticity fitting. The Young's modulus is determined by fitting an extended FD curve to avoid the influence of plastic deformation, with the assumption of the Derjaguin-Müller-Toporov model and a spherical tip shape. A Poisson ratio of 0.3 was used in the calculation as a reasonable assumption for amyloid fibrils. ${ }^{62,63}$ A filter was applied to distinguish amyloid fibrils and substrate on the QI-AFM images, and further calculation and statistical analysis were realized in OriginPro (OriginLab Corporation, MA, USA).

\section{ASSOCIATED CONTENT}

\section{SI Supporting Information}

The Supporting Information is available free of charge at https://pubs.acs.org/doi/10.1021/acsnano.0c07570.

Schematics of the experimental setup, CFD analysis on the oscillation-induced hydrodynamics, ThT fluorescence assay, AFM maps and morphology of the $\alpha$-Syn protein in solution before incubation, extraction of morphological features of amyloid fibrils, statistical distribution of the length and the average height of mature fibrils, fibril heterogeneity in the static S-bubble condition, analysis on the flexibility of amyloid fibrils, nanomechanical characterization of the amyloid fibrils, and the formation of multistranded ribbon (PDF)

\section{AUTHOR INFORMATION}

\section{Corresponding Authors}

Francesco S. Ruggeri - Department of Chemistry, University of Cambridge, Cambridge CB2 1EW, U.K.; Laboratory of Organic Chemistry and Laboratory of Physical Chemistry, Wageningen University, 6708 WE Wageningen, The Netherlands; (1) orcid.org/0000-0002-1232-1907; Email: simone.ruggeri@wur.nl

Giovanni Dietler - Laboratory of Physics of Living Matter, École Polytechnique Fédérale de Lausanne (EPFL), CH-1015 Lausanne, Switzerland; 이이.org/0000-0002-78070880; Email: giovanni.dietler@epfl.ch

\section{Authors}

Jiangtao Zhou - Laboratory of Physics of Living Matter, École Polytechnique Fédérale de Lausanne (EPFL), CH-1015

Lausanne, Switzerland; (1) orcid.org/0000-0003-4248-2207

Leonardo Venturelli - Laboratory of Physics of Living Matter, École Polytechnique Fédérale de Lausanne (EPFL), CH-1015 Lausanne, Switzerland; 이이.org/0000-00029708-8170

Ludovic Keiser - Laboratory of Fluid Mechanics and Instabilities, École Polytechnique Fédérale de Lausanne (EPFL), CH-1015 Lausanne, Switzerland

Sergey K. Sekatskii - Laboratory of Physics of Living Matter, École Polytechnique Fédérale de Lausanne (EPFL), CH-1015 Lausanne, Switzerland; (1) orcid.org/0000-0003-4706-0652

François Gallaire - Laboratory of Fluid Mechanics and Instabilities, École Polytechnique Fédérale de Lausanne (EPFL), CH-1015 Lausanne, Switzerland

Sandor Kasas - Laboratory of Physics of Living Matter, École Polytechnique Fédérale de Lausanne (EPFL), CH-1015 Lausanne, Switzerland 
Giovanni Longo - Istituto di Struttura della Materia, CNR, 00133 Roma, Italy; (1) orcid.org/0000-0003-2434-2155

Tuomas P. J. Knowles - Department of Chemistry and Cavendish Laboratory, University of Cambridge, Cambridge CB2 1EW, U.K.; $\odot$ orcid.org/0000-0002-7879-0140

Complete contact information is available at:

https://pubs.acs.org/10.1021/acsnano.0c07570

\section{Author Contributions}

J.Z., F.S.R., S.S., and G.D. designed the research; J.Z., L.V., G.L., and F.S.R. performed experiments; L.K., F.G., and S.K. contributed simulations; J.Z., F.S.R., L.V., T.P.J.K., and G.D. analyzed data; J.Z., F.S.R., L.V., and G.D. wrote the manuscript. All authors edited the manuscript.

\section{Author Contributions}

${ }^{\mathrm{I}} \mathrm{L}$. Venturellia and L. Keiserb contributed equally.

\section{Notes}

The authors declare no competing financial interest.

\section{ACKNOWLEDGMENTS}

We thank Raffaele Mezzenga from Laboratory of Food and Soft Material (FSM) in ETH Zurich, Christian Heinis and Xudong Kong from Laboratory of Therapeutic Proteins and Peptides (LPPT), and Hilal Lashuel from Laboratory of Molecular and Chemical Biology of Neurodegeneration (LMNN) in EPFL for their advice and facilities. We acknowledge the support from the Swiss National Science Foundation Grants (200021_162767) and Darwin College.

\section{REFERENCES}

(1) Chiti, F.; Dobson, C. M. Protein Misfolding, Functional Amyloid, and Human Disease. Annu. Rev. Biochem. 2006, 75 (1), 333-366.

(2) Knowles, T. P. J.; Vendruscolo, M.; Dobson, C. M. The Amyloid State and Its Association with Protein Misfolding Diseases. Nat. Rev. Mol. Cell Biol. 2014, 15 (6), 384.

(3) Eisenberg, D.; Jucker, M. The Amyloid State of Proteins in Human Diseases. Cell 2012, 148 (6), 1188-1203.

(4) Simone Ruggeri, F.; Habchi, J.; Cerreta, A.; Dietler, G. AFMBased Single Molecule Techniques: Unraveling the Amyloid Pathogenic Species. Curr. Pharm. Des. 2016, 22 (26), 3950-3970.

(5) Wei, G.; Su, Z.; Reynolds, N. P.; Arosio, P.; Hamley, I. W.; Gazit, E.; Mezzenga, R. Self-Assembling Peptide and Protein Amyloids: From Structure to Tailored Function in Nanotechnology. Chem. Soc. Rev. 2017, 46 (15), 4661-4708.

(6) Ling, S.; Kaplan, D. L.; Buehler, M. J. Nanofibrils in Nature and Materials Engineering. Nature Reviews Materials 2018, 3 (4), 18016.

(7) Knowles, T. P. J.; Mezzenga, R. Amyloid Fibrils as Building Blocks for Natural and Artificial Functional Materials. Adv. Mater. 2016, 28 (31), 6546-6561.

(8) Usov, I.; Adamcik, J.; Mezzenga, R. Polymorphism Complexity and Handedness Inversion in Serum Albumin Amyloid Fibrils. ACS Nano 2013, 7 (12), 10465-10474.

(9) Adamcik, J.; Mezzenga, R. Amyloid Polymorphism in the Protein Folding and Aggregation Energy Landscape. Angew. Chem., Int. Ed. 2018, 57 (28), 8370-8382.

(10) Annamalai, K.; Gührs, K.-H.; Koehler, R.; Schmidt, M.; Michel, H.; Loos, C.; Gaffney, P. M.; Sigurdson, C. J.; Hegenbart, U.; Schönland, S.; Fändrich, M. Polymorphism of Amyloid Fibrils in Vivo. Angew. Chem., Int. Ed. 2016, 55 (15), 4822-4825.

(11) Seuring, C.; Verasdonck, J.; Ringler, P.; Cadalbert, R.; Stahlberg, H.; Böckmann, A.; Meier, B. H.; Riek, R. Amyloid Fibril Polymorphism: Almost Identical on the Atomic Level, Mesoscopically Very Different. J. Phys. Chem. B 2017, 121 (8), 1783-1792.
(12) Li, B.; Ge, P.; Murray, K. A.; Sheth, P.; Zhang, M.; Nair, G.; Sawaya, M. R.; Shin, W. S.; Boyer, D. R.; Ye, S.; Eisenberg, D. S.; Zhou, Z. H.; Jiang, L. Cryo-EM of Full-Length $\alpha$-Synuclein Reveals Fibril Polymorphs with a Common Structural Kernel. Nat. Commun. 2018, 9 (1), 3609.

(13) Fitzpatrick, A. W. P.; Falcon, B.; He, S.; Murzin, A. G.; Murshudov, G.; Garringer, H. J.; Crowther, R. A.; Ghetti, B.; Goedert, M.; Scheres, S. H. W. Cryo-EM Structures of Tau Filaments from Alzheimer's Disease. Nature 2017, 547 (7662), 185-190.

(14) Paravastu, A. K.; Leapman, R. D.; Yau, W.-M.; Tycko, R. Molecular Structural Basis for Polymorphism in Alzheimer's $\beta$ Amyloid Fibrils. Proc. Natl. Acad. Sci. U. S. A. 2008, 105 (47), 1834918354.

(15) Zhang, S.; Andreasen, M.; Nielsen, J. T.; Liu, L.; Nielsen, E. H.; Song, J.; Ji, G.; Sun, F.; Skrydstrup, T.; Besenbacher, F.; Nielsen, N. C.; Otzen, D. E.; Dong, M. Coexistence of Ribbon and Helical Fibrils Originating from HIAPP20-29 Revealed by Quantitative Nanomechanical Atomic Force Microscopy. Proc. Natl. Acad. Sci. U. S. A. 2013, 110 (8), 2798-2803.

(16) Reynolds, N. P.; Adamcik, J.; Berryman, J. T.; Handschin, S.; Zanjani, A. A. H.; Li, W.; Liu, K.; Zhang, A.; Mezzenga, R. Competition between Crystal and Fibril Formation in Molecular Mutations of Amyloidogenic Peptides. Nat. Commun. 2017, 8 (1), 1338.

(17) Lewandowski, J. R.; van der Wel, P. C. A.; Rigney, M.; Grigorieff, N.; Griffin, R. G. Structural Complexity of a Composite Amyloid Fibril. J. Am. Chem. Soc. 2011, 133 (37), 14686-14698.

(18) Usov, I.; Mezzenga, R. Correlation between Nanomechanics and Polymorphic Conformations in Amyloid Fibrils. ACS Nano 2014, 8 (11), 11035-11041.

(19) Ruggeri, F. S.; Longo, G.; Faggiano, S.; Lipiec, E.; Pastore, A.; Dietler, G. Infrared Nanospectroscopy Characterization of Oligomeric and Fibrillar Aggregates during Amyloid Formation. Nat. Commun. 2015, 6, 7831.

(20) Ruggeri, F. S.; Adamcik, J.; Jeong, J. S.; Lashuel, H. A.; Mezzenga, R.; Dietler, G. Influence of the $\beta$-Sheet Content on the Mechanical Properties of Aggregates during Amyloid Fibrillization. Angew. Chem. 2015, 127 (8), 2492-2496.

(21) Ruggeri, F. S.; Vieweg, S.; Cendrowska, U.; Longo, G.; Chiki, A.; Lashuel, H. A.; Dietler, G. Nanoscale Studies Link Amyloid Maturity with Polyglutamine Diseases Onset. Sci. Rep 2016, 6 (1), 111.

(22) Wheeler, M. A.; Jaronen, M.; Covacu, R.; Zandee, S. E. J.; Scalisi, G.; Rothhammer, V.; Tjon, E. C.; Chao, C.-C.; Kenison, J. E.; Blain, M.; Rao, V. T. S.; Hewson, P.; Barroso, A.; Gutiérrez-Vázquez, C.; Prat, A.; Antel, J. P.; Hauser, R.; Quintana, F. J. Environmental Control of Astrocyte Pathogenic Activities in CNS Inflammation. Cell 2019, 176 (3), 581-596.

(23) Rellán-Álvarez, R.; Lobet, G.; Dinneny, J. R. Environmental Control of Root System Biology. Annu. Rev. Plant Biol. 2016, 67 (1), 619-642.

(24) Morris, R. J.; Eden, K.; Yarwood, R.; Jourdain, L.; Allen, R. J.; MacPhee, C. E. Mechanistic and Environmental Control of the Prevalence and Lifetime of Amyloid Oligomers. Nat. Commun. 2013, 4 (1), $1-8$

(25) Jordens, S.; Riley, E. E.; Usov, I.; Isa, L.; Olmsted, P. D.; Mezzenga, R. Adsorption at Liquid Interfaces Induces Amyloid Fibril Bending and Ring Formation. ACS Nano 2014, 8 (11), 1107111079 .

(26) Nielsen, L.; Khurana, R.; Coats, A.; Frokjaer, S.; Brange, J.; Vyas, S.; Uversky, V. N.; Fink, A. L. Effect of Environmental Factors on the Kinetics of Insulin Fibril Formation: Elucidation of the Molecular Mechanism. Biochemistry 2001, 40 (20), 6036-6046.

(27) Kurouski, D.; Lu, X.; Popova, L.; Wan, W.; Shanmugasundaram, M.; Stubbs, G.; Dukor, R. K.; Lednev, I. K.; Nafie, L. A. Is Supramolecular Filament Chirality the Underlying Cause of Major Morphology Differences in Amyloid Fibrils? J. Am. Chem. Soc. 2014, 136 (6), 2302-2312. 
(28) Pellarin, R.; Schuetz, P.; Guarnera, E.; Caflisch, A. Amyloid Fibril Polymorphism Is under Kinetic Control. J. Am. Chem. Soc. 2010, 132 (42), 14960-14970.

(29) Adamcik, J.; Castelletto, V.; Bolisetty, S.; Hamley, I. W.; Mezzenga, R. Direct Observation of Time-Resolved Polymorphic States in the Self-Assembly of End-Capped Heptapeptides. Angew. Chem., Int. Ed. 2011, 50 (24), 5495-5498.

(30) Campioni, S.; Carret, G.; Jordens, S.; Nicoud, L.; Mezzenga, R.; Riek, R. The Presence of an Air-Water Interface Affects Formation and Elongation of $\alpha$-Synuclein Fibrils. J. Am. Chem. Soc. 2014, 136 (7), 2866-2875.

(31) Jean, L.; Lee, C. F.; Vaux, D. J. Enrichment of Amyloidogenesis at an Air-Water Interface. Biophys. J. 2012, 102 (5), 1154-1162.

(32) Leiske, D. L.; Shieh, I. C.; Tse, M. L. A Method To Measure Protein Unfolding at an Air-Liquid Interface. Langmuir 2016, 32 (39), 9930-9937.

(33) Grigolato, F.; Colombo, C.; Ferrari, R.; Rezabkova, L.; Arosio, P. Mechanistic Origin of the Combined Effect of Surfaces and Mechanical Agitation on Amyloid Formation. ACS Nano 2017, 11 (11), 11358-11367.

(34) Zhou, J.; Ruggeri, F. S.; Zimmermann, M. R.; Meisl, G.; Longo, G.; Sekatskii, S. K.; Knowles, T. P. J.; Dietler, G. Effects of Sedimentation, Microgravity, Hydrodynamic Mixing and Air-Water Interface on $\alpha$-Synuclein Amyloid Formation. Chem. Sci. 2020, 11 (14), 3687-3693.

(35) Liu, J.; Tian, M.; Shen, L. Surface Effects on the Degree of Twist in Amyloid Fibril Structures. Chem. Commun. 2020, 56 (21), 3147-3150.

(36) Dobson, J.; Kumar, A.; Willis, L. F.; Tuma, R.; Higazi, D. R.; Turner, R.; Lowe, D. C.; Ashcroft, A. E.; Radford, S. E.; Kapur, N.; Brockwell, D. J. Inducing Protein Aggregation by Extensional Flow. Proc. Natl. Acad. Sci. U. S. A. 2017, 114, 201702724.

(37) Bekard, I. B.; Asimakis, P.; Bertolini, J.; Dunstan, D. E. The Effects of Shear Flow on Protein Structure and Function. Biopolymers 2011, 95 (11), 733-745.

(38) Thomas, C. R.; Geer, D. Effects of Shear on Proteins in Solution. Biotechnol. Lett. 2011, 33 (3), 443-456.

(39) Liu, L.; Qi, W.; Schwartz, D. K.; Randolph, T. W.; Carpenter, J. F. The Effects of Excipients on Protein Aggregation during Agitation: An Interfacial Shear Rheology Study. J. Pharm. Sci. 2013, 102 (8), 2460-2470.

(40) Vogel, V.; Sheetz, M. Local Force and Geometry Sensing Regulate Cell Functions. Nat. Rev. Mol. Cell Biol. 2006, 7 (4), 265275 .

(41) Hardy, J.; Cullen, K. Amyloid at the Blood Vessel Wall. Nat. Med. 2006, 12 (7), 756-757.

(42) Smith, E. E.; Greenberg, S. M. Beta-Amyloid, Blood Vessels and Brain Function. Stroke 2009, 40 (7), 2601-2606.

(43) Ruggeri, F. S.; šneideris, T.; Vendruscolo, M.; Knowles, T. P. J. Atomic Force Microscopy for Single Molecule Characterisation of Protein Aggregation. Arch. Biochem. Biophys. 2019, 664, 134-148.

(44) Adamcik, J.; Jung, J.-M.; Flakowski, J.; Rios, P. D. L.; Dietler, G.; Mezzenga, R. Understanding Amyloid Aggregation by Statistical Analysis of Atomic Force Microscopy Images. Nat. Nanotechnol. 2010, 5 (6), 423.

(45) Jeong, J. S.; Ansaloni, A.; Mezzenga, R.; Lashuel, H. A.; Dietler, G. Novel Mechanistic Insight into the Molecular Basis of Amyloid Polymorphism and Secondary Nucleation during Amyloid Formation. J. Mol. Biol. 2013, 425 (10), 1765-1781.

(46) Borst, A. G.; van Loon, J. J. W. A. Technology and Developments for the Random Positioning Machine, RPM. Microgravity Sci. Technol. 2009, 21 (4), 287-292.

(47) Becker, J. L.; Souza, G. R. Using Space-Based Investigations to Inform Cancer Research on Earth. Nat. Rev. Cancer 2013, 13 (5), $315-327$.

(48) Dinarelli, S.; Longo, G.; Dietler, G.; Francioso, A.; Mosca, L.; Pannitteri, G.; Boumis, G.; Bellelli, A.; Girasole, M. Erythrocyte's Aging in Microgravity Highlights How Environmental Stimuli Shape Metabolism and Morphology. Sci. Rep. 2018, 8 (1), 1-12.
(49) Wuest, S. L.; Stern, P.; Casartelli, E.; Egli, M. Fluid Dynamics Appearing during Simulated Microgravity Using Random Positioning Machines. PLoS One 2017, 12 (1), No. e0170826.

(50) Kapur, S.; Mohan, S.; Baylink, D. J.; Lau, K.-H. W. Fluid Shear Stress Synergizes with Insulin-Like Growth Factor-I (IGF-I) on Osteoblast Proliferation through Integrin-Dependent Activation of IGF-I Mitogenic Signaling Pathway. J. Biol. Chem. 2005, 280 (20), 20163-20170.

(51) Ruggeri, F. S.; Benedetti, F.; Knowles, T. P. J.; Lashuel, H. A.; Sekatskii, S.; Dietler, G. Identification and Nanomechanical Characterization of the Fundamental Single-Strand Protofilaments of Amyloid $\alpha$-Synuclein Fibrils. Proc. Natl. Acad. Sci. U. S. A. 2018, 115 (28), 7230-7235.

(52) vandenAkker, C. C.; Engel, M. F. M.; Velikov, K. P.; Bonn, M.; Koenderink, G. H. Morphology and Persistence Length of Amyloid Fibrils Are Correlated to Peptide Molecular Structure. J. Am. Chem. Soc. 2011, 133 (45), 18030-18033.

(53) Witz, G.; Rechendorff, K.; Adamcik, J.; Dietler, G. Conformation of Circular DNA in Two Dimensions. Phys. Rev. Lett. 2008, 101 (14), 148103.

(54) Ruggeri, F. S.; Flagmeier, P.; Kumita, J. R.; Meisl, G.; Chirgadze, D. Y.; Bongiovanni, M. N.; Knowles, T. P. J.; Dobson, C. M. The Influence of Pathogenic Mutations in $\alpha$-Synuclein on Biophysical and Structural Characteristics of Amyloid Fibrils. ACS Nano 2020, 14, 5213.

(55) Foderà, V.; Pagliara, S.; Otto, O.; Keyser, U. F.; Donald, A. M. Microfluidics Reveals a Flow-Induced Large-Scale Polymorphism of Protein Aggregates. J. Phys. Chem. Lett. 2012, 3 (19), 2803-2807.

(56) Campioni, S.; Bagnani, M.; Pinotsi, D.; Lecinski, S.; Rodighiero, S.; Adamcik, J.; Mezzenga, R. Interfaces Determine the Fate of Seeded $\alpha$-Synuclein Aggregation. Adv. Mater. Interfaces 2020, 7 (11), 2000446.

(57) Grigolato, F.; Arosio, P. Synergistic Effects of Flow and Interfaces on Antibody Aggregation. Biotechnol. Bioeng. 2020, 117 (2), 417-428.

(58) Adamcik, J.; Mezzenga, R. Adjustable Twisting Periodic Pitch of Amyloid Fibrils. Soft Matter 2011, 7 (11), 5437.

(59) Knowles, T. P. J.; De Simone, A.; Fitzpatrick, A. W.; Baldwin, A.; Meehan, S.; Rajah, L.; Vendruscolo, M.; Welland, M. E.; Dobson, C. M.; Terentjev, E. M. Twisting Transition between Crystalline and Fibrillar Phases of Aggregated Peptides. Phys. Rev. Lett. 2012, 109 (15), 158101.

(60) Volpatti, L. R.; Vendruscolo, M.; Dobson, C. M.; Knowles, T. P. J. A Clear View of Polymorphism, Twist, and Chirality in Amyloid Fibril Formation. ACS Nano 2013, 7 (12), 10443-10448.

(61) Mikhaylov, A.; Sekatskii, S. K.; Dietler, G. DNA Trace: A Comprehensive Software for Polymer Image Processing. J. Adv. Microsc. Res. 2013, 8 (4), 241-245.

(62) Xu, Z.; Paparcone, R.; Buehler, M. J. Alzheimer's A $\beta(1-40)$ Amyloid Fibrils Feature Size-Dependent Mechanical Properties. Biophys. J. 2010, 98 (10), 2053-2062.

(63) del Mercato, L. L.; Maruccio, G.; Pompa, P. P.; Bochicchio, B.; Tamburro, A. M.; Cingolani, R.; Rinaldi, R. Amyloid-Like Fibrils in Elastin-Related Polypeptides: Structural Characterization and Elastic Properties. Biomacromolecules 2008, 9 (3), 796-803. 